The Hanus solution, iodine monobromide, dissolved in glacial acetic acid, has many advantages as a reagent in this determination on account of its stability and permanence. Using this solution for determining the iodine number of the cod liver oils examined, a much wider variation was found than is suggested in the U. S. Pharmacopoeia. This is due, however, to the same reason as was noted in the discussion of the variation observed for the specific gravity and other constants.

In conclusion, the writer would say that the standards and tests of the U. S. Pharmacopoeia which are satisfactory for Norwegian oils are not applicable to the American oils, which are liable to show more variation, and the color tests as laid down are not characteristic of cod liver oil but only of fish liver oils. Further, the nitric acid test is liable to give misleading results with many pure cod liver oils of American origir.

\title{
THE PREPARATION OF ALDEHYDE-FREE ETHYL ALCO- HOL FOR USE IN OIL AND FAT ANALYSIS.
}

BY FREDERICK I, DUNLAP.

Received January 2, 1906.

ONE of the most common reagents in the analysis of oils and fats is alcoholic potassium hydroxide. In the preparation of this reagent, it is necessary to remove the aldehyde from the alcohol before the potassium hydroxide is dissolved in it, otherwise the solution obtained is more or less dark colored, according to the amount of aldehyde present. There are cases in which the dark color of the alcoholic potassium hydroxide offers no obstacle, but where the solution is a standard, it is necessary that it be water-white. Especially is this true where a titration is made in a solution which is itself dark colored and in which the end-point is, at best, not of the sharpest. Cases of this kind are by no means infrequent in determining the saponification value of oils and fats.

In the preparation of aldehyde-free alcohol, it is desirable that the method be fairly rapid, and that the yield be as nearly quantitative as possible. The most common method is to treat the alcohol with potassium hydroxide (ro grams per liter) and let stand for ten days to two weeks; or, in lieu of this, to boil this alcoholic solution for three hours. The dark colored solution 
is then distilled and the distillate used in the preparation of the alcoholic potassium hydroxide. It has been my experience that under the conditions as given, the acetaldehyde is not completely resinified and that the first fractions must be rejected. In fact, I have found the same to be true in cases where much more than Io grams of potassium hydroxide were added to each liter of alcohol and where such solutions stood for several months before distillation.

Waller ${ }^{1}$ has proposed the treatment of alcohol with potassium permanganate, letting the solution stand until the permanganate has all been reduced and then fractioning with a column, rejecting those fractions which turn yellow with potassium hydroxide. This method is, at best, a slow one, as the fractioning is to be conducted so that no more than $50 \mathrm{cc}$. of distillate are obtained in twenty minutes. Then too, the yield of aldehydefree alcohol is reduced by the amount that must be rejected on account of its turning yellow with alkali.

Bell ${ }^{2}$ has suggested a method in which $500 \mathrm{cc}$. of alcohol are treated with 25 grams of potassium hydroxide and the resulting solution boiled with 250 grams of lard or other easily saponifiable fat, after which the alcohol is distilled from the resulting soap and unsaponified oil and used for preparing alcoholic potassium hydroxide solutions. Bell states that he obtains $450 \mathrm{cc}$. of good alcohol by this process.

$\mathrm{Kitt}^{3}$ has objected to Waller's method on the ground of the yield, and has suggested that better results may be obtained by fractioning the alcohol without the use of potassium permanganate, rejecting those portions that turn yellow with potassium hydroxide. I may say that Kitt's method likewise may be reasonably objected to on the same ground that Kitt objected to Waller's. For example, I found that a sample of commercial 95 per cent. alcohol on fractioning gave but $40-50$ per cent. of an aldehyde-free product, whereas if a column was used the yield was increased to 75 per cent., yet this is far from satisfactory.

In a recent article in the Berichte, ${ }^{4} \mathrm{~L}$. W. Winkler gives a method for preparing pure absolute ethyl alcohol, in which the

1 This Journal, I I, I24 ( I889).

2 J. Soc. Chem. Ind. I2, 236 ( 1893 ).

${ }^{3}$ Chem. Rev. Fett Harz Ind, x I, I73 (Ig04).

4 Ber. 28, 3612 (1905). 
aldehyde is removed by means of silver oxide. Winkler prepared the silver oxide by precipitating silver nitrate with an excess of alkali, washing, and drying at ordinary temperatures. In order to make the silver oxide as eff ective as possible, it must be finely divided, so Winkler was obliged to grind the dry silver oxide in a mortar with a small amount of alcohol before adding it to the main lot which was to be purified. The alcohol and oxide were allowed to stand for several days with frequent shaking. The alcohol so treated was found to be absolutely aldehydefree.

The above method of Winkler suggested to me the use of silver oxide in preparing aldehyde-free alcohol for use in oil and fat analysis and, with certain variations, gave a method which leaves little to be desired so far as ease of execution, rapidity, and yield are concerned. The principal variation from Winkler's method lies in the fact that the silver oxide is precipitated in the alcohol, in a finely divided condition, being therefore more active and rapid in its action than when prepared apart and then added. The method I have adopted is as follows:

Dissolve 1.5 grams of silver nitrate in about $3 \mathrm{cc}$. of water and add it to a liter of 95 per cent. alcohol in a glass-stoppered cylinder, then mix thoroughly. Dissolve 3 grams of potassium hydroxide (by alcohol) in ro to $15 \mathrm{cc}$. of warm alcohol and, after cooling, pour it slowly into the alcoholic silver nitrate solution, but do not shake. The silver oxide is precipitated in a very finely divided condition and slowly distributes itself throughout the contents of the cylinder. Let stand quietly over night or until the precipitated silver oxide has completely settled. Either siphon off the clear supernatant liquid from the sediment or filter, and distil. None of the distillate need be rejected for it will all give with potassium hydroxide a water-white solution. Moreover, due to the excess of alkali used, the distillate is perfectly neutral and therefore serviceable for the determination of the acid value of oils, or for any other purpose where a neutral alcohol is necessary.

The amount of silver nitrate given in the method above may or may not furnish sufficient silver oxide for rapid and complete oxidation of the aldehyde, for this will depend on the amount of aldehyde in the alcohol treated, but the amount of silver nitrate may be varied to suit the need for it. However, the proportions 
given have proved ample for any alcohol which $I$ have had occasion as yet to make aldehyde-free.

If for any reason it may be desirable to avoid the use of water in dissolving the silver nitrate, this salt may be powdered and then dissolved in the 95 per cent. alcohol, and then the alkali added to this.

The above method has several advantages over the procedure of Winkler. There is a great saving of time in precipitating the silver oxide in the alcohol rather than preparing it apart and then utilizing it. The great advantage gained by this is that the oxide is thrown down in such a minute state of subdivision and hence in the very best condition for a rapid and complete oxidation of the aldehyde. If the cylinder be shaken after the addition of the alkali, the silver oxide gathers in flocks which rapidly settle to the bottom of the cylinder, and are thus much less effective than the finely divided and slowly settling precipitate. Shaking is therefore to be avoided.

It should be said in justice to Winkler, that the method outlined above would have been impracticable for his purpose, for he was preparing pure absolute alcohol and the introduction of water into the so-called absolute alcohol which he used as his starting point would not have been feasible. Furthermore, silver nitrate is practically insoluble in absolute alcohol so that it was necessary to prepare the silver oxide separately. For fat analysis, the presence of a few per cent. of water in the alcohol offers no disadvantages.

The method as outlined above is not applicable to methyl alcohol.

I wish to thank Mr. Lorin $\mathrm{H}$. Bailey for his aid in carrying out a part of the experimental work on this subject.

CHEMTCAL LABORATORY, UNIVERSITY OF MICHIGAN, December 27,1905 .

\section{NOTES.}

Note on the Condensation of Succinylosuccinic Esterswith Amidines. -In a recent paper in this Journal (27, 1302) by us, the condensation of succinylosuccinic acid diethyl ester with acetamidine and the product resulting therefrom are described. Before this work was undertaken the literature was examined 